The final publication is available at Springer via https://doi.org/10.1007/ 978-3-319-09873-9_27. 


\title{
Workflow Scheduling on Federated Clouds
}

\author{
Juan J. Durillo ${ }^{1}$ and Radu Prodan ${ }^{1}$ \\ University of Innsbruck, Innsbruck, Austria \\ juan, radu@dps.uibk.ac.at
}

\begin{abstract}
Federated Clouds, or the orchestration of multiple Cloud services for fulfilling applications' requirements, is receiving increasing attention. Despite their many advantages, federated Clouds also present some downsides since different services may reside in different geographically located areas. This paper focuses on evaluating the advantages and disadvantages of using a federation of Clouds for executing scientific workflows. It evaluates a wide range of different workflow types with different requirements in terms of computation and communication (produced and consumed data), and discusses which kind of workflow applications can benefit from a Cloud federation and how.
\end{abstract}

\section{Introduction}

Workflows emerged as a popular paradigm used by scientists and engineers to model scientific and industrial applications. Workflow applications are usually executed on parallel and distributed systems to minimize their completion time or makespan. A challenging task here is to determine the "optimal" mapping or schedule of the workflow tasks onto the available resources that produces the shortest makespan, a well-known NP-complete problem.

Nowadays, Cloud computing is becoming attractive for companies and institutions to access to parallel and distributed resources. Despite the promise of theoretically infinite number of resources, data centers acting as Cloud providers have limited capacity to be shared among many clients. In overloaded situations, a provider infrastructure may not be large enough to accommodate all customers' requests, which negatively impacts its popularity. To mitigate this problem, several providers limit the maximum number of instances that can be simultaneously used by a single user, for example to 20 by Amazon EC2. In response to this, federated Clouds [12] aim to coordinate (federate) the Cloud infrastructure of several institutions, offering access to their services and, therefore, significantly increasing the resources available to an application. A Cloud federation can be viewed as a marketplace where providers sell and buy ondemand computational capacity and offer it transparently to their customers. In this case, if a single provider does not have enough resources for accommodating a customer request, it can buy the missing capacity from the marketplace.

Federated Clouds bring new opportunities when using commercial Clouds, as different providers may offer resources with different performance and pricing models. In such situations, a customer may be interested in scheduling lowpriority tasks on the slow resources offered by a cheap provider, and high-priority 
critical tasks on the expensive fast resources offered by a high-performance provider. Scheduling a workflow application becomes therefore a multi-objective optimisation problem with at least two in-conflict criteria, makespan and financial cost, to which a single solution does not exist. In fact, the solution to this kind of problems consists of a set of tradeoff solutions between the conflicting criteria, known as Pareto front. Moreover, federated Clouds pose additional drawbacks limiting the range of applications that can benefit from them. For example, resources belonging to different providers may be located in different areas connected via best-effort Internet, which is particularly problematic in the context of data-intensive applications. In addition, companies may impose restrictions over sensitive data that must stay within the frontiers of a single institution, limiting optimisation opportunities. In this situations, it is not clear whether federated Clouds are an appealing alternative for workflow applications.

In this paper we investigate the potential of a federation of Clouds for multicriteria workflow scheduling. We tackle the problem from the point of view of a broker coordinating the services of two federated Clouds with different performance and price models: Amazon EC2 and GoGrid ${ }^{1}$. We analyse how that federation can be used to schedule workflow applications with different shapes (degree of parallelism), different number of activities, and different requirements in terms of computation and communication. The contributions of this paper are threefold: (1) we describe the multi-objective workflow scheduling problem on federated Clouds using the Multi-Objective Heterogeneous Earliest Finish Time (MOHEFT) algorithm; (2) we analyse whether federated Clouds improve the execution of workflow application; and (3) we analyse of the tradeoff between the workflow execution and its financial cost on federated Clouds.

The rest of the manuscript is organized as follows. Next section contains related work. In Section 3 we describe our architecture and research problem, followed by the Cloud-aware MOHEFT scheduling algorithm in Section 4. Sections 5 and 6 describe the experimental setup and the obtained results. Finally, Section 7 contains the conclusions and the future work.

\section{Related Work}

Previous attempts to multi-objective workflow scheduling consisted in transforming the problem into a mono-objective optimization one using user preferences [3, 9,11 , imposing constraints over the optimisation criteria [16,8], or optimising single objectives individually [4]. More recently, several approaches computing the whole set of tradeoff solutions emerged grouped in two main lines: (1) genetic algorithms-based techniques for optimizing makespan and cost [23], makespan and energy consumption [13], or makespan, cost and reliability [18]; and (2) list-based heuristics for optimizing makespan and cost or makespan $[5,6]$ and energy consumption [7]. Only few of these works targeted workflwo scheduling on Clouds and none of them considers Cloud federations.

\footnotetext{
${ }^{1}$ http://www.gogrid.com/products/cloud-hosting
} 
While most related research deals with the placement of Virtual Machines (VM) onto Physical Machines (PM) [14, 15, 20, 21], scheduling of tasks onto VMs has been scarcely studied on the context of a Cloud federation. In this sense, [10] analyses and proposes several heuristics for task scheduling onto resources belonging to the same provider but geographically located in different areas. Though not purely a federation of Clouds, the authors address a similar problem to the one arising in a federated system. The authors in [17] present a semantic architecture to build schedulers for federated Cloud with emphasis on the system architecture with no special focus to the optimality of the scheduler. None of these approaches consider a purely multi-objective formulation of the problem.

\section{Model}

We describe in this section the architecture, workflow, and resource model underneath our approach, together with the makespan and financial cost objectives.

\subsection{Architecture}

Fig. 1 depicts our system architecture whose main component is the broker coordinating resources belonging to different Cloud providers. This broker receives a workflow application as input and is in charge of computing several schedule solutions trading off makespan and financial cost. Once these tradeoff solutions are computed, it selects a schedule out of them, usually user-guided and biased by external conditions. Solution selection mechanisms are out of the scope of this paper, which focuses on computing an optimal and representative set of tradeoff solutions. Once a solution has been selected, the broker is in charge of resource provisioning and deploying the workflow activities for execution. In order to compute the tradeoff schedules, the broker needs to know the workflow, the available resource types, and how to compute the makespan and financial cost entailed by different schedules.

\subsection{Workflow Model}

We model a workflow as a directed acyclic graph: $W=(A, D)$ consisting of $n$ activities $A=\bigcup_{i=1}^{n}\left\{A_{i}\right\}$, interconnected through control flow and data flow dependencies; $D=\left\{\left(A_{i}, A_{j}\right.\right.$, Data $\left.\left._{i j}\right) \mid\left(A_{i}, A_{j}\right) \in A \times A\right\}$, where Data $_{i j}$ represents the size of the data which needs to be transferred from activity $A_{i}$ to activity $A_{j}$. We use $\operatorname{pred}\left(A_{i}\right)=\left\{A_{k} \mid\left(A_{k}, A_{i}\right.\right.$, Data $\left.\left._{k i}\right) \in D\right\}$ to denote the predecessor set of activity $A_{i}$, (i.e. activities to be completed before starting $A_{i}$ ). We assume that the computational workload of every activity $A_{i}$ is known and is given by the number of machine instructions to be executed.

\subsection{Resource Model}

The broker assumes that our hardware platform consists of a set of $m$ heterogeneous resources $R=\cup_{j=1}^{m} R_{j}$, which can be of any type as our case studies in 

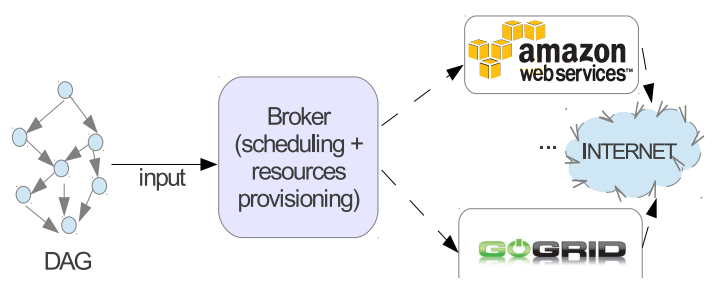

Fig. 1: System architecture.

\begin{tabular}{|l|c|c|c|}
\hline Instance & $\begin{array}{c}\text { Speed } \\
{[\text { GFLOPS] }]}\end{array}$ & $\begin{array}{c}\text { Price } \\
{[\$ / h]}\end{array}$ & $\begin{array}{c}\text { GFLOPS/ } \\
\$\end{array}$ \\
\hline \hline \multicolumn{4}{|c|}{ Amazon EC2 Instances } \\
\hline m1. small & 2.0 & 0.1 & 19.6 \\
m1.large & 7.1 & 0.4 & 17.9 \\
m1.xlarge & 11.4 & 0.8 & 14.2 \\
c1.medium & 3.9 & 0.2 & 19.6 \\
c1.xlarge & 50.0 & 0.8 & 62.5 \\
\hline \hline \multicolumn{4}{|c|}{ GoGrid Instances } \\
\hline GG.large & 8.8 & 0.16 & 46.4 \\
GG.xlarge & 28.1 & 0.76 & 37.0 \\
\hline
\end{tabular}

Fig. 2: EC2 and GoGrid performance and prices.

this paper Amazon EC2 and GoGrid. In particular, we consider in this paper the five Amazon EC2 resources and the two GoGrid instances analysed in [2] and summarized in Table 2. For a given resource $R_{j}$ of a certain type, we know its average performance measured in GFLOPs and its price per hour of computation. The final price of a schedule is based the resources' usage and the data stored and transferred. This price depends on four components: (1) price per hours of resource usage $P E_{R_{i}},(2)$ price per $\mathrm{MB}$ of data storage $P S_{R_{i}},(3)$ price per $\mathrm{MB}$ of data received $P I_{R_{i}}$, and (4) price per $\mathrm{MB}$ of data sent $P O_{R_{i}}$. The prices of these components depend on the Cloud provider. As mentioned before, Cloud providers may impose some constraints too. While in theory a user can access an infinite pool of resources, most providers restrict this number to a maximum of $N$ instances that can be simultaneously acquired. These $N$ resources can be of any type and do not have to be kept invariant during execution. We use $\operatorname{sched}\left(A_{i}\right)$ to denote the resource on which the task $A_{i}$ is scheduled for execution.

\subsection{Makespan}

To compute the makespan, it is necessary to define the execution time $t_{\left(A_{i}, R_{j}\right)}$ of an activity $A_{i}$ on a resource $R_{j}=\operatorname{sched}\left(A_{i}\right)$ as the sum of the time required for transferring the biggest input data from any $A_{p} \in \operatorname{pred}\left(A_{p}\right)$ and the time required to execute $A_{i}$ on $R_{j}$ :

$$
t_{\left(A_{i}, R_{j}\right)}=\max _{A_{p} \in \operatorname{pred}\left(A_{i}\right)}\left\{\frac{\text { Data }_{p i}}{b_{p j}}\right\}+\frac{\operatorname{workload}\left(A_{i}\right)}{s_{j}},
$$

where $D a t a_{p i}$ is the size of the data to be transferred between $A_{p}$ and $A_{i}, b_{p j}$ is the bandwidth between the resource where task $A_{p}$ was executed and the resource $R_{j}$ (corresponding to the bandwidth of the local network in case both resources belong to the same provider, or to the Internet connection in case they belong to different providers), workload $\left(A_{i}\right)$ represents the length of the task $A_{i}$ in machine instructions, and $s_{j}$ the speed of the resource $R_{j}$ in number of machine instructions per second (see second column of Table 2). Next, we can compute the completion time $T_{A_{i}}$ of activity $A_{i}$ considering the execution time 
of itself and its predecessors:

$$
T_{A_{i}}= \begin{cases}t_{\left(A_{i}, \text { sched }\left(A_{i}\right)\right)}, & \text { pred }\left(A_{i}\right)=\emptyset \\ \max _{A_{p} \in \operatorname{pred}\left(A_{i}\right)}\left\{T_{A_{p}}+t_{\left(A_{i}, \operatorname{sched}\left(A_{i}\right)\right)}\right\}, & \text { pred }\left(A_{i}\right) \neq \emptyset .\end{cases}
$$

We define the makespan as the maximum completion time of all activities:

$$
T_{W}=\max _{i \in[1, n]}\left\{T_{\left(A_{i}, \text { sched }\left(A_{i}\right)\right)}\right\} .
$$

\subsection{Financial Cost}

The financial cost for executing an activity depends on two terms: the computation cost $C^{(\mathrm{comp})}$ and the cost of data transfer and storage $C^{(\text {data) }}$. We assume that all providers charge the customers using an hourly based model (i.e. per hour of computation). We define $C_{\left(A_{i}, R_{j}\right)}^{(\text {data }}$ as the cost of the data transfers $\operatorname{In}\left(A_{i}\right)$ and $\operatorname{Out}\left(A_{i}\right)$ and storage $\operatorname{Data}\left(A_{i}\right)$ resulting from executing activity $A_{i}$ on resource $R_{j}$ :

$$
C_{\left(A_{i}, R_{j}\right)}^{(\text {data })}=\operatorname{Data}\left(A_{i}\right) \cdot t_{\left(A_{i}, R_{j}\right)} \cdot P S_{R_{i}}+\operatorname{In}\left(A_{i}\right) \cdot P I_{R_{i}}+\text { Out }\left(A_{i}\right) \cdot P O_{R_{i}},
$$

In defining the cost $C_{R_{j}}^{(c o m p)}$ of using a resource $R_{j}$, we assume that for each task $A_{i}$ executed on $R_{j}$ we record two timestamps: $t_{A_{i}}^{(\text {start })}$ when the activity starts and $t_{A_{i}}^{(e n d)}$ when the activity finishes its execution. We consider without loss of generality that the times for transferring the input $\operatorname{In}\left(A_{i}\right)$ and the output data Out $\left(A_{i}\right)$ are included in the interval between $t_{A_{i}}^{\text {(start })}$ and $t_{A_{i}}^{(\text {end })}$.

Let us consider now the set of all $p$ activities scheduled on resource $R_{j}$ denoted as $\left\{J_{1}, \ldots, J_{p}\right\}$, where $p<n$ and sched $\left(J_{i}\right)=R_{j}, i \in[1, p]$, sorted based on their start timestamp: $t_{J_{1}}^{(\text {start })}<\ldots<t_{J_{p}}^{(\text {start })}$. Based on this ordering, we cluster these activities in $q \leq p$ different groups $G_{k}^{(j)}, 1 \leq k \leq q$. All activities in one group are executed consecutively without releasing the resource. After the activity with the largest start timestamp in the group completes, the resource is released.

We construct the first group $G_{1}^{(j)}=\left\{J_{1}, \ldots, J_{r}\right\}, r \leq p$ following three rules:

1. The first activity $J_{1}$ belongs to the first group: $J_{1} \in G_{1}^{(j)}$;

2. Every activity $J_{i} \in G_{1}^{(j)}, 2 \leq i \leq r$, starts before the current leased hour expires and before the machine is released:

$$
t_{J_{i}}^{(\text {start })}<t_{J_{1}}^{(\text {start })}+\left\lceil\frac{t_{J_{i-1}}^{(\text {end })}-t_{J_{1}}^{(\text {start })}}{3600}\right\rceil \cdot 3600 .
$$

We convert the total time of using a resource to hours by dividing it by 3600 and using the ceiling operator. This equation guarantees a contiguous resource allocation of activities within one hour slot; 
3. The next activity not part of the first group $J_{r+1} \notin G_{1}^{(j)}, r+1 \leq p$, starts after the last hour of computation elapses and the resource is released:

$$
t_{J_{1}}^{(\text {start })}+\left\lceil\frac{t_{J_{r}}^{(\text {end })}-t_{J_{1}}^{(\text {start })}}{3600}\right\rceil \cdot 3600<t_{J_{r+1}}^{(\text {start })} .
$$

Successive groups are built until the last activity $J_{p}$ has been assigned to one group. The second group $G_{2}^{(j)}$ is constructed in the same way starting from $J_{r+1}$ instead of $J_{1}$, and similarly for the rest of the groups. Once all groups have been created, we define the cost $C_{R_{j}}^{(c o m p)}$ of using the resource $R_{j}$ as the number of hours required for executing all groups multiplied by the cost per hour:

$$
C_{R_{j}}^{(c o m p)}=P E_{R_{j}} \cdot \sum_{k=1}^{q}\left\lceil\frac{\sum_{A_{i} \in G_{R_{j}}^{(k)}} t_{\left(A_{i}, R_{j}\right)}}{3600}\right\rceil .
$$

The cost of executing the workflow $W=(A, D)$ is the sum of the cost of all $m$ the used resources and the cost for transferring and storing the data:

$$
C_{W}=\sum_{j=1}^{m} C_{R_{j}}^{(c o m p)}+\sum_{\left(A_{i}, A_{j}, D a t a_{i j}\right) \in D} C_{\left(A_{i}, R_{j}\right)}^{(\text {data }} .
$$

\section{Cloud-Aware MOHEFT Algorithm}

We employ a method for computing the tradeoff solutions as an extension of the MOHEFT [6] algorithm customised for dealing with the characteristics of federated Cloud environments. MOHEFT is a list-based heuristic which extends the HEFT [19] algorithm to consider multiple simultaneous criteria. MOHEFT, summarized in Algorithm 1, requires the the instance types offered by all Cloud providers, the maximum number of resources that can be simultaneously rented from each provider $\boldsymbol{N}$, and the desired number of tradeoff solutions $K$.

Firstly, MOHEFT ranks the tasks in the workflow using the B-rank metric and creates a set $S$ of $K$ empty schedules (lines 2 and 3 ). Afterwards, it iterates over the list of tasks and extends every solution in $S$ by mapping the next task onto different possible instances. For every task, the algorithm builds a list of possible resources where the task can be executed, either by reusing an instance already assigned to a previous task, or by acquiring a new instance (lines 10 and 14, respectively). This list is used for building new schedules that also consider the current task. The newly produced schedules are stored in a temporary set $S^{\prime}$, initially empty. After each iteration, $S^{\prime}$ replaces $S$ before the next task in the list is considered. Obviously, this strategy results in an exhaustive search if we do not include any restrictions. To avoid this, MOHEFT saves only the best $K$ tradeoff solutions from the temporary set $S^{\prime}$ to the set $S$, selected based on the objective functions and the diversity of the set, i.e., how different these solutions are (see [6]). To deal with the restriction on the maximum number of 


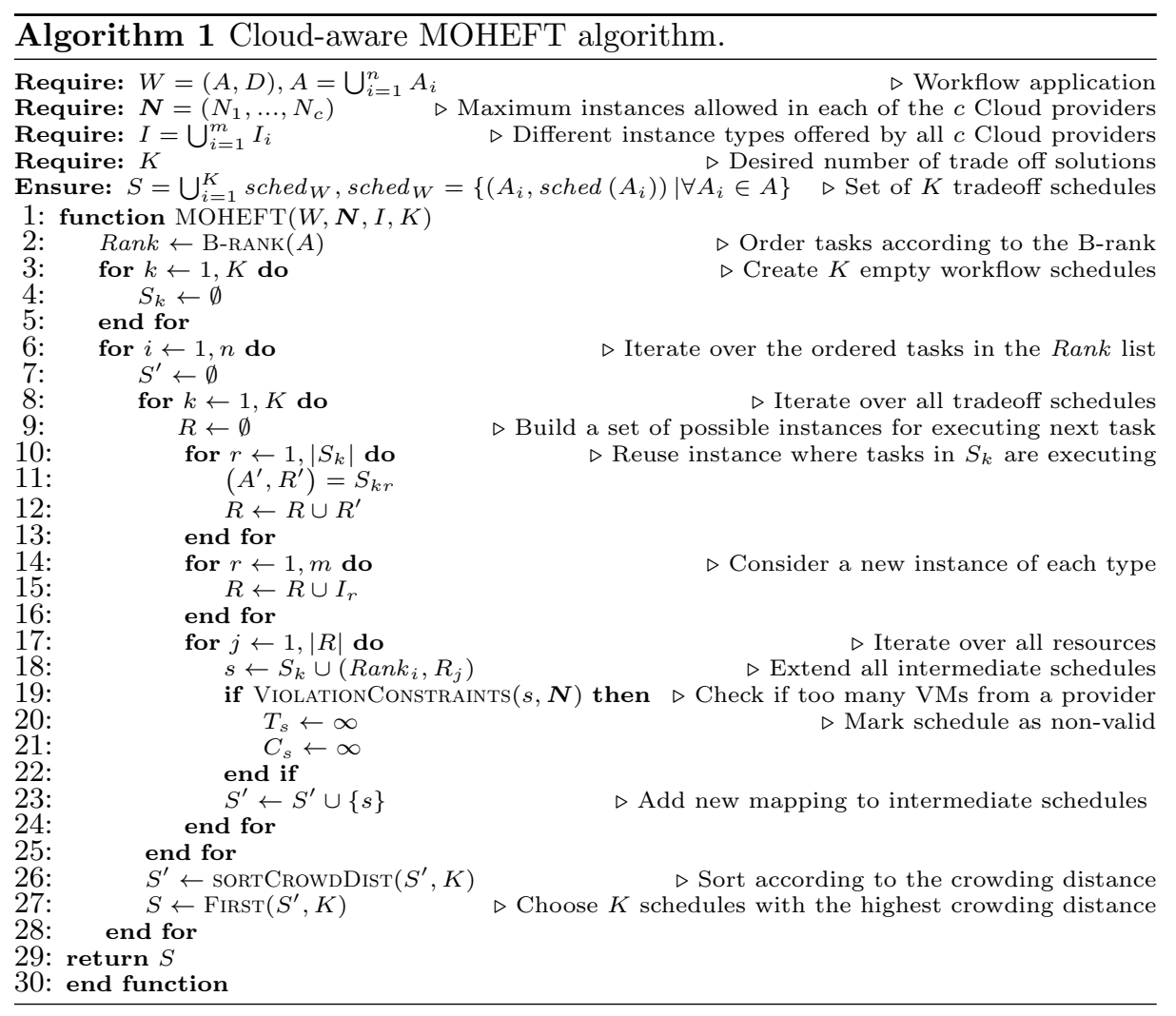

instances that can be simultaneously rented from a provider, MOHEFT discards any schedule that violates this constraint (line 19) by setting its financial costs and makespan to infinite. This way, the partial solution will be always worse (dominated) than any other schedule and will be discarded in line 26 .

\section{Experimental Setup}

We describe in this section the workflow and resource setups used in evaluating our approach.

\subsection{Workflow Applications}

We consider in our evaluation two types of workflow applications: syntheticallygenerated and real-world.

We generated the synthetic workflows as described in [22] considering two kinds of shapes: Type-1 workflows with many independent activities sharing one successor and one predecessor, and Type-2 workflows where most tasks have different successors and predecessors. Type-1 workflows represent applications 
which can clearly benefit from a high number of resources since many activities can be executed in parallel. In Type-2 workflows, the number of parallel activities is lower than in the former case, hindering but not preventing the benefit of parallel execution. In both cases, we consider workflow instances with a number of activities between 100 and 1000. We generated the length of each activity using a Gaussian distribution with the execution time of around 10 seconds on an average single core instance. For both types, we considered three classes with different data requirements: Low where each activity produces/consumes around 10MB of data, Medium producing/consuming around 100MB, and High produceing/consuming around 1GB.

The Persitence of Vision Raytracer (POV-Ray) [1] application is a free tool for creating three dimensional graphics used by scientists in biochemistry, medicine or architecture visualisation. It consists of rendering a set of images/frames, merging them, and storing in a resulting a file. The number of frames to merge determines the number of activities in the workflow. The volume of data consumed by this application also depends on the number of frames which have to be transferred to the merger activity after being rendered. We consider here three instances of this application rendering 512, 1024, and 4096 frames.

\subsection{Resources}

We carried out the experiments in a federated infrastructure with two IaaS Cloud providers: Amazon EC2 and GoGrid. We use for modelling the workflows' makespan the average performance in millions of floating point operations per second (GFLOPs) of five different instance types of Amazon EC2 and three instances of GoGrid, as reported the Iosup et al. in [2] after extensive benchmark experimentation (see Table 2). We assume that the resources of the same provider are connected using a local 1000mbps network and the different providers are connected using a $150 \mathrm{mbps}$ wide area network. We consider that a user can simultaneously rent 20 instances from both Amazon EC2 and GoGrid, which can be of any of the types summarized in Table 2. Although these limits can be usually extended upon request, we intend in this paper evaluate how MOHEFT can deal with the default configurations.

\section{Experimental Results}

In this section, we analyse the schedules computed by MOHEFT for each type of workflow when using a federation of EC2 and GoGrid Cloud and a maximum of $K=10$ tradeoff solutions. These tradeoff solutions will be graphically depicted in Figures 3, 4, and 5, showing for each of solution (numbered $0-9$ on the horizontal axis) the makespan and cost normalized in the interval $[0,1]$. This normalisation is done using the maximum and minimum values within the Pareto front for both the makespan and financial cost. These graphs also show the percentage of instances simultaneously used from the maximum allowed by each provider. This information is showed in form of bar charts, where the height of 


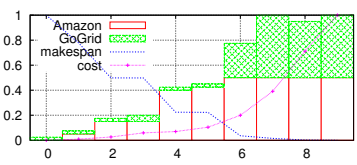

(a) Low data class.

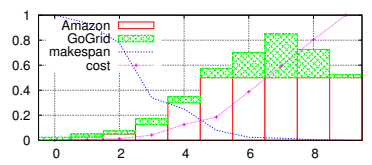

(b) Medium data class.

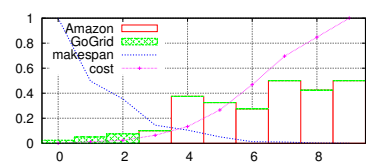

(c) High data class.

Fig. 3: Normalized Type-1 workflow tradeoff schedules.

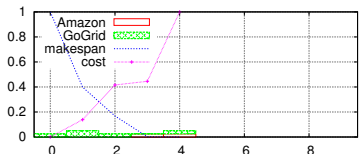

(a) Low data class.

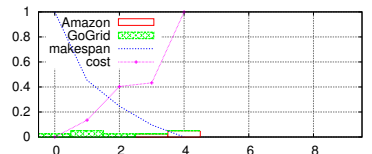

(b) Medium data class.

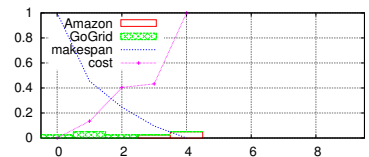

(c) High data class.

Fig. 4: Normalized Type-2 workflow tradeoff schedules.

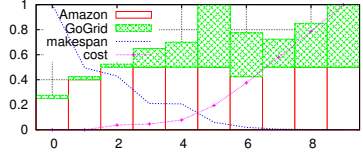

(a) 512 frames.

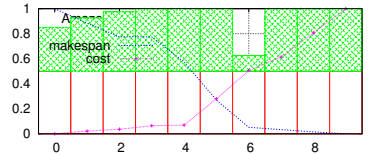

(b) 1024 frames.

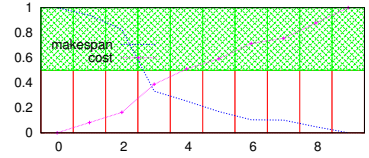

(c) 4096 frames.

Fig. 5: Normalized POV-Ray workflow tradeoff schedules.

each bar indicates the percentage normalised in the $[0,0.5]$ interval. We use a total of 40 resources (20 from each provider) as explained before, therefore, a bar with a height of 0.5 indicates that 20 provider machines have been used. We use two different bar textures to differentiate between the two considered providers. In all the experiments of this section, graphs depict the information for workflows consisting of 1000 activities; experiments with a different number of activities have shown the same behavior but have been omitted here due to space limitations.

\subsection{Type-1 Workflows}

Fig. 3 summarizes the results obtained for workflows Type-1. We focus first our analysis on the advantages of using the Cloud federation and comment afterwards on the makespan and cost of the computed solutions. The three graphs show that the benefits of using a federation of Clouds decreases with the volume of the data managed by the application. When the data volume is low, the workflow makespan can be further decreased by considering the joined use of services from the federation. We observe this behavior in Fig. 3a, where the three tradeoff schedules with smallest makespan (labelled $7,8,9$ ) uses all available machines 
provided by the federation (the percentage of machines used from both providers is 0.5 ). As long as the data volume increases, aggregating resources from different providers does not reduce the makespan, despite the fact that number of independent activities in the workflow is high. In particular, when the data volume is medium, the percentage of machines used from the federation decreased and never receased the maximum of 40 . Finally, when the data volume is high, the federated Cloud does not bring any advantage and the computed schedules only use machines of one or the other provider. Regarding the makespan and financial cost, we observe in all cases that a schedule with an overhead of less than $10 \%$ with respect to the shortest computed makespan dramatically increases the costs. For example, the difference between the makespan of the schedules labelled 6 and 9 is smaller than $5 \%$, while the difference in financial costs are up to $80 \%$ (see Fig. 3a). A deeper analysis of the results also reveals that cheap solutions rarely consider federated resources and rather use resources from a single provider. A possible explanation for this behavior is the hourly based price model offered by the providers, cheap solutions trying to increase resource utilisation instead of launching simultaneous instances.

\subsection{Type-2 Workflows}

Fig. 4 depicts the results obtained for Type-2 workflows. In all the evaluated scenarios, MOHEFT produced less than $K=10$ tradeoff schedules which indicates that the tradeoff between makespan and financial cost is lower for this kind of workflows than for those of Type-1. Regarding to the used resources, only few instances are required to execute this kind of workflows as a consequence of the lower number of activities which can be executed in parallel. Therefore, the use of a Cloud federation does not bring any benefit in this situation. The analysis of the makespan versus cost reports in this case the same the behavior as before: reducing the makespan overhead to less than $10 \%$ over the shortest one implies a strong economical investment, while schedules with more than $10 \%$ overhead imply a small price fraction.

\subsection{POV-Ray}

As explained before, each activity of the POV-Ray workflow renders a frame and transfers it to a final activity which merges all the frames and stores them into a file. Each frame is of around 1MB in size; therefore, the POV-Ray application can be considered as a Type-1 workflow and expects to achieve benefits from using a federation of Clouds. As summarized in Fig. 5, it is obvious that the federation helps in reducing the makespan of this application. The three evaluated cases show that the higher the number of activities, the higher the benefit from the federation. This result is a consequence of the high degree of parallelism showed by this workflow application and the low volume of data required. 


\section{Conclusions and Future Work}

In this paper we tackled the problem of multi-objective workflow scheduling from the perspective of a Cloud federation and presented a Cloud-aware of the multi-objective HEFT algorithm, called MOHEFT. We analyse the potential of MOHEFT to schedule workflow applications with different properties: workflow shape, size, and amount of data to be transferred among workflow activities. Experimental results using Amazon EC2 and GoGrid as independent providers illustrated that federated Clouds can help in shortening the makespan for workflow applications which do not require transferring large amounts of data among activities. In situations when data transfers dominate the computation time, the workflow does not benefit from a federation of Clouds and performs better in a single provider configuration. In future work we will extend the analysis for other real-world applications and a wider set of Cloud providers. We will also analyse extensions to MOHEFT to better deal with federated Clouds and workflows dealing with big data problems.

\section{References}

1. http://www. povray.org/.

2. I. Alexandru, S. Ostermann, M. Yigitbasi, R. Prodan, T. Fahringer, and D. Epema. Performance analysis of cloud computing services for many-tasks scientific computing. IEEE Transactions onf Parallel and Distributed Systems, 2010.

3. I. Assayad, A. Girault, and H. Kalla. A bi-criteria scheduling heuristics for distributed embedded systems under reliability and real-time constraints. In International Conference on Dependable Systems and Networks, DSN'04, Firenze, Italy, June 2003. IEEE.

4. K. Bessai, S. Youcef, A. Oulamara, C. Godart, and S. Nurcan. Bi-criteria workflow tasks allocation and scheduling in cloud computing environments. In Proceedings of the 2012 IEEE Fifth International Conference on Cloud Computing, CLOUD '12, Washington, DC, USA, 2012. IEEE Computer Society.

5. L.-C. Canon and Emmanuel. Mo-greedy: an extended beam-search approach for solving a multi-criteria scheduling problem on heterogeneous machines. International Heterogeneity in Computing, 2011.

6. J. Durillo, H. Fard, and R. Prodan. Moheft: A multi-objective lilst-based method for workflow scheduling. In 4th IEEE International Conference on Cloud Computing Technology and Science, December 2012.

7. J. J. Durillo, V. Nae, and R. Prodan. Multi-objective workflow scheduling: An analysis of the energy efficiency and makespan tradeoff. In CCGRID, 2013.

8. H. Fard, R. Prodan, J. Barrionuevo, and T. Fahringer. A multi-objective approach for workflow scheduling in heterogeneous environments. In Cluster, Cloud and Grid Computing (CCGrid), 2012 12th IEEE/ACM International Symposium on, may 2012.

9. S. K. Garg, R. Buyya, and H. J. Siegel. Scheduling parallel applications on utility grids: time and cost trade-off management. In Proceedings of the ThirtySecond Australasian Conference on Computer Science - Volume 91, ACSC '09, Darlinghurst, Australia, Australia, 2009. Australian Computer Society, Inc. 
10. S. K. Garg, C. S. Yeo, A. Anandasivam, and R. Buyya. Environment-conscious scheduling of hpc applications on distributed cloud-oriented data centers. J. Parallel Distrib. Comput., 71(6), June 2011.

11. M. Hakem and F. Butelle. Reliability and scheduling on systems subject to failures. In Proceedings of the 2007 International Conference on Parallel Processing, ICPP '07, Washington, DC, USA, 2007. IEEE Computer Society.

12. T. Kurze, M. Klems, D. Bermbach, A. Lenk, S. Tai, and M. Kunze. Cloud Federation. In Proceedings of the 2nd International Conference on Cloud Computing, GRIDs, and Virtualization (CLOUD COMPUTING 2011). IARIA, Sept. 2011.

13. M. Mezmaz, N. Melab, Y. Kessaci, Y. Lee, E.-G. T. albi, A.Y.Zomaya, and D. Tuyttens. A parallel bi-objective hybrid metaheuristic for energy-aware s cheduling for cloud computing systems. Journal of Parallel and Distributed Computing, (71), 2011.

14. L. Rao, X. Liu, and W. Liu. Minimizing electricity cost: Optimization of distributed internet data centers in a multi-electricity-market environment. In In Proc. of INFOCOM, 2010.

15. S. Ren, Y. He, and F. Xu. Provably-efficient job scheduling for energy and fairness in geographically distributed data centers. In Proceedings of the 2012 IEEE 32nd International Conference on Distributed Computing Systems, ICDCS '12, Washington, DC, USA, 2012. IEEE Computer Society.

16. R. Sakellariou, H. Zhao, E. Tsiakkouri, and M. D. Dikaiakos. Scheduling workflows with budget constraints. In in Integrated Research in Grid Computing, S. Gorlatch and M. Danelutto, Eds.: CoreGrid series. Springer-Verlag, 2007.

17. I. Santana-Perez and M. S. Perez-Hern'ndez. A semantic scheduler architecture for federated hybrid clouds. 2012 IEEE Fifth International Conference on Cloud Computing, 0, 2012.

18. A. K. M. K. A. Talukder, M. Kirley, and R. Buyya. Multiobjective differential evolution for scheduling workflow applications on global grids. Evolution, 21(13), 2009.

19. H. Topcuoglu, S. Hariri, and M.-Y. Wu. Performance-effective and low-complexity task scheduling for heterogeneous computing. Parallel and Distributed Systems, IEEE Transactions on, 13(3), mar 2002.

20. R. Urgaonkar, U. C. Kozat, K. Igarashi, and M. J. Neely. Dynamic resource allocation and power management in virtualized data centers. In NOMS. IEEE, 2010.

21. Y. Yao, L. Huang, A. Sharma, L. Golubchik, and M. Neely. Power cost reduction in distributed data centers: A two time scale approach for delay tolerant workloads. IEEE Transactions on Parallel and Distributed Systems, 99(PrePrints), 2013.

22. J. Yu, R. Buyya, and K. Ramamohanarao. Workflow scheduling algorithms for grid computing. In F. Xhafa and A. Abraham, editors, Metaheuristics for Scheduling in Distributed Computing Environments. Springer Berlin, 2008.

23. J. Yu, M. Kirley, and R. Buyya. Multi-objective planning for workflow execution on grids. In Proceedings of the 8th IEEE/ACM International Conference on Grid Computing, GRID '07, Washington, DC, USA, 2007. IEEE Computer Society. 